\title{
Exodontia chair positions: A brief overview
}

\author{
Ashvini Kishor Vadane ${ }^{*}$, Janardan Bhagwat Garde ${ }^{2}$, Alka Dattatray Sherkhane ${ }^{3}$ \\ ${ }^{1,3}$ Senior Lecturer, ${ }^{2}$ Professor and HOD, ${ }^{1,2}$ M.A. Rangoonwala College of Dental Sciences and Research Centre, Pune, \\ Maharashtra, ${ }^{3}$ Shri Yashwantrao Chavan Memorial Medical \& Rural Development Foundation's Dental College \& Hospital, \\ Ahmednagar, Maharashtra, India
}

\section{*Corresponding Author: Ashvini Kishor Vadane}

Email: drashvinivadane@gmail.com

\begin{abstract}
Exodontia is a branch of dentistry. Exodontia deals with the therapeutic extraction of tooth from its normal anatomic position, i.e., alveolar socket. aim of this short article is to discuss chair positions to be followed by clinicians.
\end{abstract}

Keywords: Chair position.

\section{Introduction}

The painless removal of the whole tooth or tooth root with minimum trauma to the investing tissues, so that wound heals uneventfully. [As per Geoffrey L. Howe]

Exodontia is a minor surgical procedure that involves principles of surgery as well as principles of physics. Dental surgeons should apply these principles appropriately to perform extractions with less complications. The use of controlled force is important for extraction of an erupted tooth. Excessive force can damage surrounding vital structures like soft tissues and alveolar bone. It also increases intraoperative \& postoperative complications. Operator's excessive haste can also increase patient's anxiety. Operator's gentle tissue handling is necessary for better tissue healing. Operator should use appropriate as well as sharp and well cared instruments for successful exodontia. Successful exodontia depends on a proper knowledge based technique and operator's skill. Operator should have knowledge of basic anatomy of maxilla \& mandible and individual tooth anatomy for performing successful extractions.

\section{Exodontia is of two types as follows}

1. Intra-alveolar extraction.

2. Transalveolar extraction.

\section{Intra-alveolar extraction}

It is also known as "closed extraction" or "conventional extraction". In case of an erupted tooth, this type of extraction is performed by expanding bony alveolar socket. Dental extraction forceps, dental elevators are used for performing this type of extraction and principles of lever, wedge and wheel \& axle are applied.

\section{Trans-alveolar extraction}

It is also known as "open extraction". It is a minor surgical procedure. This procedure is the therapeutic extraction of impacted tooth. In this procedure, access is gained through a surgical flap which also helps in visualizing tooth and to facilitate extraction, appropriate bone removal is also performed.

\section{Chair position in exodontia}

It is of prime importance to follow proper patient's position, operator's position and chair position for performing successful extractions. Extractions can be performed by operator either in sitting position or in standing position. Standing extractions are most commonly practiced as compared to the sitting dentistry.

\section{Patient's position}

Patient's head rest, back rest should be in a single line. Patient should sit straight.

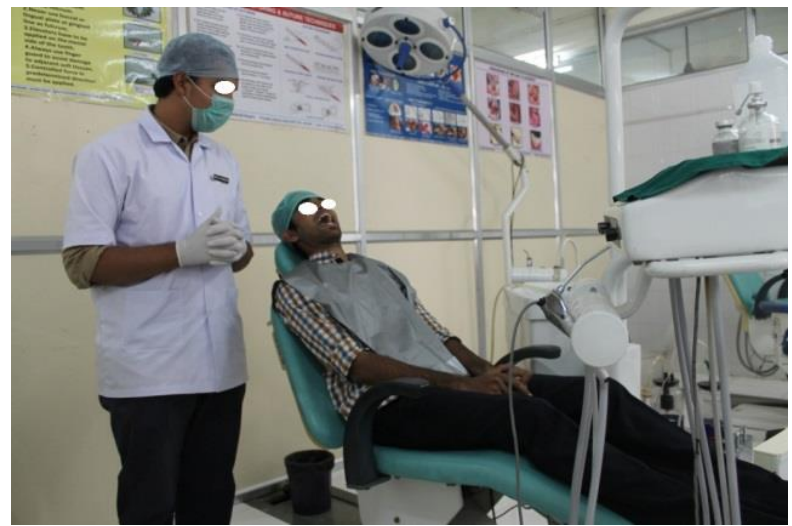

Fig. 1 a]: Chair Position for Maxillary Extractions. 


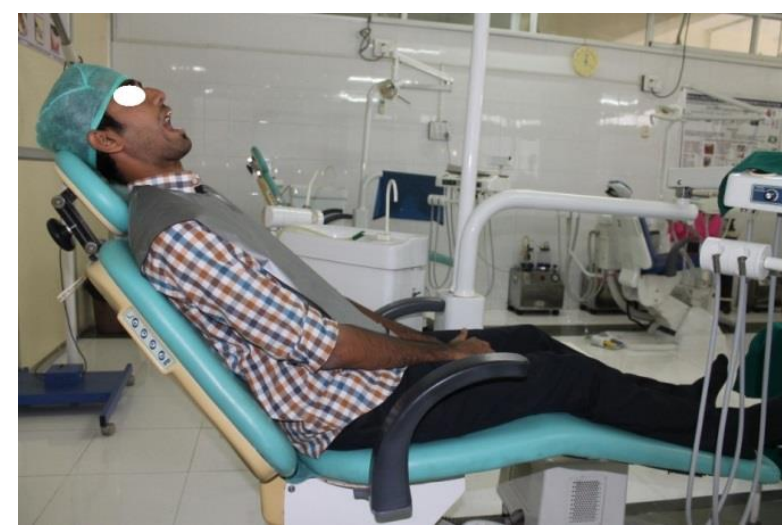

Fig. 1b]: Chair Position for Maxillary Extractions.

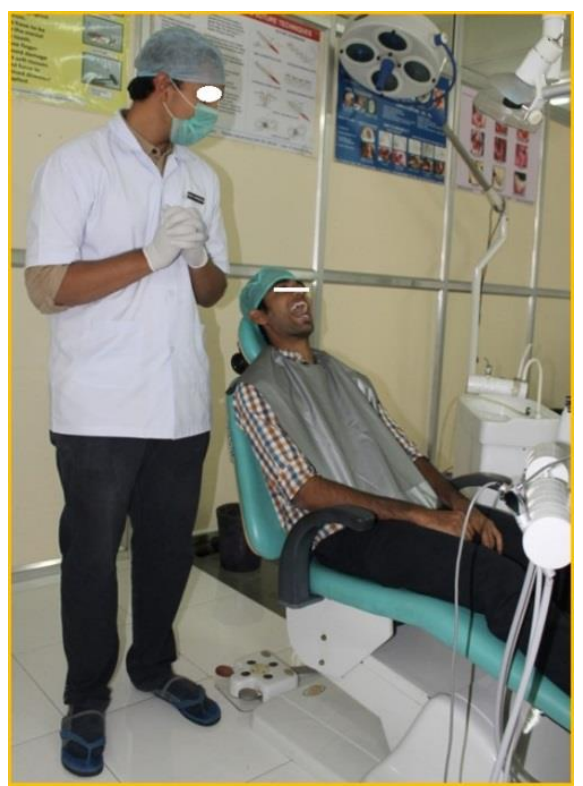

Fig. 2a]: Chair Position for Mandibular Extractions.

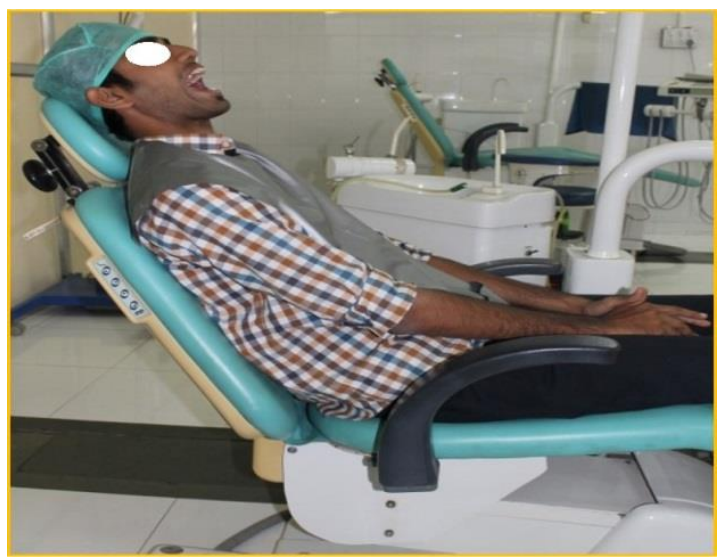

Fig. 2b]: Chair Position for Mandibular Extractions.

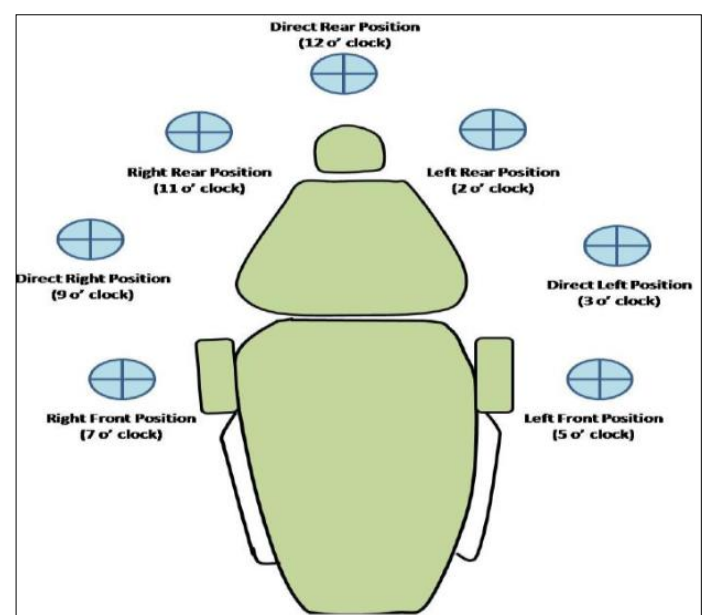

Fig. 3: Pictorial Presentation to describe chair positions in exodontia. ${ }^{2}$

\section{Operator's Position}

Operator should stand in front of the patient, for extractions in all quadrants except $4^{\text {th }}$ quadrant. Operator should stand at 7 o'clock position for performing extractions in first, second and third quadrants whereas, he should stand at 11 o'clock for performing extractions in fourth quadrant. Right handed operator should always stand on right side of the patient. Left handed operator should follow exact mirror-image positions as that of right side. While performing extractions, dentist's arms should be closed to body \& force should be applied from shoulder and wrist should be in steady position.

\section{Chair Position}

\section{Extractions of maxillary teeth}

Whenever operator plans to do maxillary teeth extractions, the chair's height should be adjusted such that the operating field should be approximately 3 inch / almost $8 \mathrm{~cm}$ below the operator's shoulder and operating field, i.e., maxillary arch should make 45 degree to 60 degree angle with the floor.

\section{Extractions of mandibular teeth}

Whenever operator plans to do mandibular teeth extractions, the chair's height should be adjusted such that the operating field, i.e., mandibular arch should be just below the operator's elbow level / at the operator's elbow level and it should be parallel to the floor.

When performing exodontia in operator's sitting position, the patient should be in supine position and operating field should be at the just below the operator's elbow level / at the operator's elbow level.

\section{Source of funding}

None. 


\section{Conflict of interest}

None.

\section{References}

1. Bhandari AJ. Dental Operator's Posture And Position. Int J Dent Health Sci 2(5).

2. Kumaresan R. The most compatible position of operator for mandibular right posterior teeth extraction. Plast Aesth Res 2016;3:257-8

3. Gadicherla S. Evaluation of a New Ergomonic Position for Operator/Clinicians for the Extraction of Mandibular Posterior Molar Teeth. J Int Oral Health 2018;10:36-9.
4. Hupp J. Textbook of "Contemporary Oral and Maxillofacial Surgery"- $7^{\text {th }}$ Edition.

5. Robinson PD. Tooth Extraction: a practical guide. Ist ed. Oxford: Wright; 2000:34-5.

How to cite: Vadane AK, Garde JB, Sherkhane AD. Exodontia chair positions: A brief overview. Int Dent $J$ Student Res 2020;8(1):37-9. 